\title{
КОМПЛЕКСНАЯ ОЦЕНКА МЕСТНЫХ БЮДЖЕТОВ РЕСПУБЛИКИ БЕЛАРУСЬ
}

\begin{abstract}
М.В. Половцев*
Предложена методика комплексной оценки местных бюджетов Республики Беларусь путем сопоставления официальной формулировки бюджета с его реальными количественными характеристиками. Полученная оценка является основой для присвоения бюджету класса соответствия. Методика просчитана на областном бюджете Брестской области на 2017 г.
\end{abstract}

Ключевые слова: местный бюджет, оценка бюджета, моделирование.

JEL-классификация: H61, H72, R51.

DOI: $10.46782 / 1818-4510-2021-3-113-124$

Материал поступил 22.02.2021 2.

Модернизация системы управления государственными финансами в Республике Беларусь тесно связана с бюджетной системой страны. В процессе ее совершенствования мониторинг достигнутых результатов должен опираться на максимально достоверную и полную информацию о текущем и/или перспективном состоянии как республиканского, так и местных бюджетов ${ }^{1}$. Наличие и грамотное использование качественного инструментария по диагностике и оценке бюджетов является важным условием для достижения целей модернизации государственной финансовой системы.

Вопросам бюджетирования государственных финансов уделяется повышенное внимание со стороны ученых и практиков. Так, российский ученый Г.Б. Поляк, рассматривая данные вопросы, предлагал интересные и прогрессивные мероприятия для совершенствования бюджетной системы страны $^{2}$. Казахский ученый Г. Мутанов

${ }^{1}$ Бюджетный Кодекс Республики Беларусь. Ст. 6, п. 2. URL: https://kodeksy-by.com/byudzhetnyj_kodeks_rb/6.htm

2 Поляк Г.Б. (Ред.). 1999. Бюджетная система России. Москва: ЮНИТИ-ДАНА. 550 с.
(2015) широко использовал математический аппарат для исследования бюджета государства. В Кыргызстане вопросами государственного бюджета занималась Н.М. Тюлюндиева (2011), в Республике Беларусь Т.В. Сорокина (1998; 2020).

Однако экспертным сообществом основное внимание уделялось теоретическим аспектам, описанию существующего порядка бюджетирования, бюджетному процессу, контролю и аудиту, анализу доходов и расходов, а также выработке предложений по совершенствованию бюджетной системы государства. В то же время оценивание государственного бюджета остается малоизученной областью․

\section{Краткий обзор международных методик по оценке государственного бюджета}

В настоящее время используются две методики по оценке бюджетов: по системе

3 Половцев М.В. 2018. Оценка эффективности управления государственными финансами. Экономика, моделирование, прогнозирование: сб. научных трудов. Вып. 12. С. 64-73; Половцев М.В. 2018. Открытость и прозрачность государственного бюджета: оценка по методике международного бюджетного партнерства. Экономический бюллетень НИЭИ Минэкономики РБ. № 11. С. 41-48.

* Половцев Максим Владимирович (optimat@tut.by), Научно-исследовательский экономический институт Министерства экономики Республики Беларусь (г. Минск, Беларусь). https://orcid.org/0000-0003-4344-3285 
$\mathrm{PEFA}^{4}$ и методологии IBР 5 . Обе эти методики были рассмотрены автором ${ }^{6}$. Общей для них является оценка прозрачности и открытости государственного бюджета, причем методология IBP полностью посвящена этому, а система PEFA лишь частично. Такое внимание к степени открытости и прозрачности бюджета не случайно. Это обусловлено тем, что «по данным международных экспертов сокращение уровня непрозрачности бюджета в среднем на один пункт равнозначно увеличению среднегодового ВВП на душу населения примерно на 1 тыс. долл. США, прямых иностранных инвестиций по отношению к ВВП - на 1 процент и сокращению инфляции на 0,5 процента» ${ }^{7}$.

Следует отметить, что необходимость стремления к прозрачности и открытости бюджетов в Республике Беларусь была осознана около двух десятилетий назад (Шимов, Александрович, Богданович, Ткачев, 2001. С. 256). Несмотря на целесообразность достижения большей прозрачности и открытости бюджета, оценка государственных бюджетов по методике IBP в Беларуси не проводилась. Оценка государственных финансов по методике, принятой в PEFA, у нас осуществлялась в 2009 и 2013 гг. на национальном уровне, т. е. местные бюджеты не оценивались.

По итогам проведенного исследования и на основе полученного отчета в 2015 г. на государственном уровне принято решение о реформировании в Республике Беларусь системы управления государственными финансами в целях обеспечения долгосрочной сбалансированности и устойчивости бюджетной системы, повышения качества управления государственными финансами. Была сформулирована Стратегия реформирования системы управления

\footnotetext{
${ }^{4}$ URL: https:// www.pefa.org

5 URL: https://www.internationalbudget.org

6 Половцев М.В. 2018. Оценка эффективности управления государственными финансами. Экономика, моделирование, прогнозирование: сб. научных трудов. Вып. 12. С. 64-73; Половцев М.В. 2018. Открытость и прозрачность государственного бюджета: оценка по методике международного бюджетного партнерства. Экономический бюллетень НИЭИ Минэкономики РБ. № 11. С. 41-48.

7 Стратегия реформирования системы управления государственными финансами Республики Беларусь. С. 29. URL: https://pravo.by/upload/docs/op/C21501080_1452286800.pdf
}

государственными финансами Республики Беларусь и предусмотрено обучение государственных служащих и специалистов для эффективного исполнения поставленных задач в настоящей стратегии.

Каждая страна обладает полным правом на свою бюджетную систему. Оценивание бюджетов разных уровней по национальной системе оценки иногда может быть более целесообразным, чем «вынужденное» следование международным стандартам. Это обуславливает актуальность разработки суверенной методики оценки государственных бюджетов в Республике Беларусь и ее практическое использование.

Исследуем возможности развития методического инструментария оценки государственных бюджетов Беларуси с целью разработки относительно простой методики оценивания местных бюджетов, дополняющей и развивающей международные методики в части прозрачности и открытости, реалистичности бюджета, а также отражающей специфику бюджетного законодательства страны. Комплексность оценки состоит в том, что элементы рассматриваются по возможности взаимоувязанно и одновременно, при этом мы получаем частные оценки, сводимые к одной интегральной оценке. Непосредственно методика комплексной оценки ориентирована на решение конкретных практических вопросов: прозрачность и доступность для понимания, уровень точности прогнозирования и итоговое соответствие законодательству страны, обеспечение сравнимости бюджетов между собой. Отличительной чертой данной методики по сравнению с вышеописанными международными методиками является использование доступного математического аппарата.

\section{Концептуальные и методологические аспекты комплексной оценки местных бюджетов}

Цель исследования - теоретическая проработка и научное обоснование методики комплексной оценки местных бюджетов. Основная задача - это разработка системы количественных показателей, соотносимых с качественными характеристиками бюджета по определенным критериям, а 
также получение на ее основе интегральной (комплексной) оценки.

Общий методологический подход к оценке местных бюджетов основывается на концептуальном допущении того, что официальное определение бюджета имеет первоочередное смысловое значение перед его фактическим воплощением, которое, по сути, является производным от законодательно утвержденной формулировки. Это значит, что любой реальный местный бюджет, в каждой своей характеристике, должен максимально полно соответствовать тому, что зафиксировано в самом определении местного бюджета. Логику такого допущения можно проверить доказательством «от противного». Так, если государственный бюджет в реальности это не то, что прописано в Бюджетном кодексе страны, то как его можно идентифицировать и где материальное воплощение официального определения? Отсутствие ответов на данные вопросы либо приводит к внутренним логическим противоречиям в системе применяемых терминов, либо требует коррекции законодательства.

Новизна методики состоит в том, что оценка бюджета напрямую зависит от уровня его соответствия официальному определению. Сущность методики - это разложение официального определения бюджета на смысловые элементы для последующего их сопоставления с реальными количественными характеристиками оцениваемого бюджета по отдельности, а затем в комплексе.

В максимально обобщенном виде можно принять, что среди источников поступлений, а также направлений расходования денежных средств местного бюджета нет таких, которые бы не отражали государственные функции и задачи национального и регионального уровней. Такое допущение представляется логичным и разумным, поскольку налоги, сборы, иные платежи в пользу государства, а также направления государственного обеспечения сами по себе связаны с вопросами внутренней государственной политики. Также можно принять, что комбинация наименований поступлений в местный бюджет и их стоимостная структура соответствуют определению «План формирования денежных средств»; сочетание наименований направлений рас- ходов и их стоимостная структура - определению «план использования денежных средств». При этом сам план должен быть максимально понятным (прозрачным и открытым) для заинтересованных сторон: общества, бизнеса, инвесторов и др.

Важно, что оценке будут подвергаться не сами направления расходования денежных средств, указанные в расходной части бюджета, а потоки финансовых ресурсов вида «доход - расход», содержащие информацию об объемах денежных средств, обеспечивающих финансирование конкретного расхода отдельным доходом. Такое расширение области оценивания позволит достичь комплексности получаемых оценок. Кроме того, предусмотрена прогнозная оценка точности плана путем сопоставления утвержденного бюджета с исполненным бюджетом.

В соответствии с принципом общего (совокупного) покрытия расходов ${ }^{8}$ можно принять, что объем поступлений из одного источника в различных пропорциях распределяется между неизвестным количеством направлений расходов; одно направление расходов обеспечивается финансированием из неизвестного количества источников поступлений, кроме целевых назначений по законодательству. Данное предположение делает доступными анализ и оценку местного бюджета относительно распределения его потоков на основе матрицы вида «доход - расход».

Центральное место в методике принадлежит матрице вида «доход - расход», в которой отражаются искомые потоки между доходами и расходами. В такой матрице распределения $X=\left(x_{i j}\right)_{m \times n}$ каждый элемент $x_{i j}$ показывает величину потока $i$-го дохода на финансирование $j$-го расхода. Индекс $m$ - это количество наименований доходов бюджета, а индекс $n$ - это количество наименований расходов бюджета. Сумма по строке - это величина по конкретному наименованию раздела дохода бюджета, а сумма по столбцу величина по конкретному наименованию вида расхода бюджета. Основное назначение матрицы $X$ - это показать текущее (плано-

\footnotetext{
${ }^{8}$ Бюджетный Кодекс Республики Беларусь. Ст. 8, п. 8. URL:https://kodeksy-by.com/byudzhetnyj_kodeks_rb/8.htm
} 
вое) распределение потоков в бюджете при его утверждении. Аналогичная матрица $Y=\left(y_{i j}\right)_{m \times n}$ содержит информацию о пропорциональном распределении потоков для исполненного бюджета.

Вычисляемое отклонение между этими двумя матрицами показывает степень точности прогнозирования - прогнозную оценку. Принимается следующее: чем больше отклонение планового состояния местного бюджета по матрице $X$ от фактического состояния местного бюджета по матрице $Y$, тем ниже его прогнозная оценка. Предполагается: чем меньше различий между утвержденным и исполненным бюджетами, тем выше точность прогнозирования на этапе планирования и одновременно выше уровень соответствия местного бюджета понятию «план использования денежных средств».
Таким образом, в результате оценки определяется, насколько план хорош - понятен, подробен, а также близок к реальности. Оценки бюджета можно сравнивать в статике и в динамике, а также с состояниями других местных бюджетов.

Следует отметить, что величины потоков «доход - расход» получаются путем математического расчета и отражают объем доходов, планируемый на финансирование конкретных направлений расходов (это справедливо как для матрицы $X$, так и для матрицы $Y$ ). В реальности фактические потоки доходов на расходы часто будут носить «замещающий» характер других потоков, которые в определенный момент времени не доступны для реализации. Тем не менее вышеописанные матрицы с математической точностью отражают те единовременно необходимые

Таблица 1

Условные обозначения показателей, используемые в методике

\begin{tabular}{|c|c|c|c|}
\hline $\begin{array}{c}\text { Условное } \\
\text { обозначение }\end{array}$ & Показатель & $\begin{array}{c}\text { Ед. } \\
\text { измерения }\end{array}$ & Примечание \\
\hline$O_{\partial}$ & $\begin{array}{l}\text { Оценка местного бюджета по уровню } \\
\text { открытости и прозрачности доходной части }\end{array}$ & Балл & - \\
\hline$O_{p}$ & $\begin{array}{l}\text { Оценка местного бюджета по уровню } \\
\text { открытости и прозрачности расходной части }\end{array}$ & Балл & - \\
\hline$O_{\phi}$ & $\begin{array}{l}\text { Оценка местного бюджета как плана } \\
\text { формирования денежных средств } \\
\end{array}$ & $\%$ & - \\
\hline$R$ & $\begin{array}{l}\text { Разрыв между оптимальным и текущим } \\
\text { состоянием доходной части местного } \\
\text { бюджета }\end{array}$ & $\%$ & $\begin{array}{l}\text { По методике определения } \\
\text { пороговых значений }\end{array}$ \\
\hline$B$ & Баланс бюджета & Руб. & $\begin{array}{l}\text { При дефиците бюджета } B \\
\text { соответствует суммарным } \\
\text { доходам, при профиците - } \\
\text { суммарным расходам, при } \\
\text { сбалансированном бюджете - } \\
\text { равенству доходов и расходов }\end{array}$ \\
\hline$D_{i}$ & $\begin{array}{l}\text { Доход бюджета по } i \text {-му наименованию } \\
\text { поступления }\end{array}$ & Руб. & Сумма по $i$-й строке \\
\hline$Z_{j}$ & $\begin{array}{l}\text { Расход бюджета по } j \text {-му наименованию } \\
\text { расхода }\end{array}$ & Руб. & Сумма по $j$-му столбцу \\
\hline$D$ & Совокупные доходы бюджета & Руб. & - \\
\hline$Z$ & Совокупные расходы бюджета & Руб. & - \\
\hline$O_{u}$ & $\begin{array}{l}\text { Оценка бюджета как плана использования } \\
\text { денежных средств }\end{array}$ & $\%$ & - \\
\hline$O_{n}$ & Прогнозная оценка бюджета & $\%$ & $\begin{array}{l}\text { Реалистичность первоначально } \\
\text { утвержденного плана }\end{array}$ \\
\hline$O_{k}$ & Комплексная оценка бюджета & & - \\
\hline$C_{o}$ & $\begin{array}{l}\text { Совокупное количество набранных баллов } \\
\text { по описательной оценке }\end{array}$ & Балл & - \\
\hline$C_{p}$ & $\begin{array}{l}\text { Совокупное количество баллов, набранных } \\
\text { по расчетной оценке }\end{array}$ & Балл & - \\
\hline
\end{tabular}

9 Половцев М.В. 2020. Моделирование местных бюджетов Республики Беларусь: пороговые значения статей и другие параметры бюджета. Экономика, моделирование, прогнозирование: сб. научных трудов. Минск: НИЭИ Министерства экономики Республики Беларусь. Вып. 14. С. 36-48.

Источник. Авторская разработка. 
объемы поступлений, без которых финансирование расходов невозможно.

\section{Методика комплексной оценки местного бюджета в Республике Беларусь}

Предлагаемая методика по комплексной оценке бюджетов (далее - методика) предназначена для анализа и оценки местных бюджетов Республики Беларусь. Она может быть использована органами государственного управления и самоуправления для совершенствования процесса бюджетирования государственных финансов.

Объект исследования - местный бюджет в Республике Беларусь.

Предмет исследования - соответствие материального воплощения местного бюджета существующему определению.

Процесс оценки местного бюджета состоит из 3 этапов:

1) описательная оценка бюджета - как общего плана с достаточным уровнем открытости и прозрачности;

2) расчетная оценка бюджета - как плана формирования денежных средств; как плана использования денежных средств с некоторой степенью точности прогноза;

3) комплексная оценка местного бюджета - на соответствие своему определению.

Перечень показателей и условные обозначения, используемые в методике, представлены в табл. 1.

Этап 1. Получение описательных оценок бюджета.

Оценка местного бюджета $O_{\partial}$ осуществляется следующим образом. В случае, если в доходной части все наименования поступлений полностью закодированы в соответствии с бюджетным классификатором ${ }^{10}$ до последнего уровня, бюджет получает 1 балл. Если же в доходной части все наименования поступлений не имеют кодировки, то бюджет получает 0 баллов, при этом не имеет значения детализация конкретных статей. Бюджет получает 0,5 балла за любое промежуточное состояние, отличное от первых двух.

Оценка местного бюджета $O_{p}$ проводится аналогично. В случае, если в расходной части все наименования направлений рас-

10 Постановление Министерства финансов Республики Беларусь от 31 декабря 2008 г. № 208 « бюджетной классификации Республики Беларусь». ходов полностью закодированы до последнего уровня, бюджет получает 1 балл. Если же в расходной части все наименования направлений расходов не имеют кодировки, то бюджет получает 0 баллов, при этом не имеет значения детализация конкретных статей. Бюджет получает 0,5 балла за любое промежуточное состояние, отличное от первых двух.

Общая описательная оценка $C_{\text {o }}$ в баллах вычисляется по формуле

$$
C_{o}=O_{\partial}+O_{p}
$$

Этап 2. Получение расчетных оценок бюджета.

Оценка местного бюджета как плана формирования денежных средств $O_{\phi}$ рассчитывается по формуле

$$
O_{\phi}=R-100 .
$$

Процесс оценки местного бюджета как плана использования денежных средств состоит из 4 шагов.

Шаг 1. Анализ бюджета на наличие расхода «Бюджетные кредиты, ссуды, займы» код 011002.

В случае, если величина такого расхода имеет отрицательное значение, необходимо произвести его исключение из дальнейшего рассмотрения. Это обусловлено тем, что отрицательная величина расхода не требует финансирования. Баланс бюджета принимается равным полученным совокупным расходам или доходам после исключения данного направления расходов.

Шаг 2. Расчет матрицы «доход - расход» $X$ - пропорциональное распределение потоков в утвержденном бюджете.

На данном шаге строится математическая модель пропорционального распределения доходов между расходами, т. е. производится поиск конкретных величин $x_{i j}$. Beличина денежных потоков вида «доход расход» рассчитывается по формуле

$$
x_{i j}=\frac{D_{i} Z_{j}}{B}, i=\overline{1, m}, j=\overline{1, n} .
$$

Следует рассматривать три случая: дефицитный бюджет, профицитный бюджет и сбалансированный бюджет с учетом результатов, полученных на шаге 1. Так, при дефицитном бюджете необходимо ввести фиктивный источник доходов $D_{m+1}$ с объемом по- 
ступлений, равным объему дефицита, для этого в матрицу «доход - расход» добавляется снизу строка с индексом $m+1$. При профицитном бюджете требуется ввести фиктивное направление расходов $Z_{n+1}$ с объемом потребности, равным объему профицита, для этого в матрицу «доход - расход» добавляется справа столбец с индексом $n+1$. При сбалансированном бюджете матрица «доход расход» остается без изменений. В табл. 2 приведена общая схема расчета потоков «доход - расход».

Шаг 3. Расчет матрицы «доход - расход» $Y-$ пропорциональное распределение потоков в исполненном бюджете.

Процесс получения матрицы $Y$ аналогичен процессу получения матрицы $X$ по формуле 3 с учетом табл. 2. В то же время следует иметь ввиду, что между утвержденным и исполненным бюджетами могут быть существенные расхождения в перечне наименований доходов или расходов с конкретными суммами. В таком случае необходимо привести обе матрицы к единообразному виду.

Шаг 4. Оценка местного бюджета как плана использования денежных средств $O_{u}$ (одновременно прогнозная оценка) вычисляется по формуле

$$
O_{u}=\sqrt{\frac{\sum_{i=1}^{m} \sum_{j=1}^{n}\left(x_{i j}-y_{i j}\right)^{2}}{\sum_{i=1}^{m} \sum_{j=1}^{n} x_{i j}^{2}}} \cdot 100 \%
$$

Количество баллов по расчетным оценкам присваивается по шкале оценки, представленной в табл. 3.
Параллельно вычисляется прогнозная оценка, показывающая точность и реалистичность планирования бюджета:

$$
O_{n}=100-O_{u}
$$

Общая расчетная оценка $C_{p}$ в баллах:

$$
C_{p}=O_{\phi}+O_{u} \text {. }
$$

Этап 3. Получение комплексной оценки местного бюджета, присвоение класса бюджету.

На данном этапе местный бюджет получает комплексную оценку, на основании которой ему присваивается классность. Комплексная оценка бюджета отражает степень его соответствия официальному определению, класс бюджета позволяет отнести местный бюджет к той или иной группе качества.

Комплексная оценка $O_{\kappa}$ определяется по формуле

$$
O_{\kappa}=\frac{\frac{C_{o}}{2}+\frac{C_{p}}{10}}{2}
$$

где 2 - максимальное количество баллов, которые в совокупности можно набрать на 1 этапе по описательным оценкам; 10 максимальное количество баллов, которые можно в совокупности набрать на 2 этапе по расчетным оценкам.

Класс бюджету присваивается на основании табл. 4.

Данная методика оценки местного бюджета имеет следующие достоинства:

- позволяет аттестовать бюджет на соответствие официальному определению;

- предоставляет количественную информацию о характеристиках бюджета;

Схема расчета потоков в матрице $X$ в зависимости от сбалансированности бюджета

Таблица 2

\begin{tabular}{|c|c|c|}
\hline $\begin{array}{c}\text { Вариант 1 } \\
\text { Дефицитный бюджет }\end{array}$ & $\begin{array}{c}\text { Вариант 2 } \\
\text { Сбалансированный бюджет }\end{array}$ & $\begin{array}{c}\text { Вариант 3 } \\
\text { Профицитный бюджет }\end{array}$ \\
\hline$x_{i j}=\frac{D_{i} Z_{j}}{B}$ & $x_{i j}=\frac{D_{i} Z_{j}}{B}$ & $x_{i j}=\frac{D_{i} Z_{j}}{B}$ \\
$x_{m+1, j}=\frac{D_{m+1} Z_{j}}{B}$ & - & $x_{i, n+1}=\frac{D_{i} Z_{n+1}}{B}$ \\
\hline
\end{tabular}

Источник. Авторская разработка.

Таблица 3

Общая шкала оценки для расчетных оценок

\begin{tabular}{|l|c|c|c|c|c|}
\hline Оценка $O_{\phi}, O_{u}, \%$ & $\leq 5$ & $\leq 10$ & $\leq 15$ & $\leq 20$ & $>20$ \\
\hline Балл & 5 & 4 & 3 & 2 & 1 \\
\hline
\end{tabular}

Таблица 4

Оценка и классность местного бюджета

\begin{tabular}{|l|c|c|c|c|}
\hline Оценка $O_{\kappa}$ & $\leq 0,6$ & $\leq 0,75$ & $\leq 0,9$ & $>0,9$ \\
\hline Класс бюджета & 4 & 3 & 2 & 1 \\
\hline
\end{tabular}

Источник. Авторская разработка.

118 Белорусский экономический журнал № $3 \bullet 2021$ 
- способствует увеличению прозрачности и открытости бюджета;

- не противоречит имеющимся международным методикам, дополняя их с учетом региональных особенностей местных бюджетов страны;

- дает представление о точности прогнозирования состояния местного бюджета - прогнозную оценку;

- не требует отвлечения значительных объемов трудовых, интеллектуальных, временных и материальных ресурсов.

В то же время данная методика содержит ряд особенностей:

- рекомендована для уровня областных бюджетов в большей степени, чем для нижестоящих бюджетов;

- использует «вложенную» методику.

Разработанная методика оценки имеет:

- научную значимость - впервые в Республике Беларусь обоснован принципиально новый концептуальный подход к оценке местных бюджетов, заключающийся в использовании официального определения бюджета в качестве тестового шаблона соответствия и обеспечивающий комплексное представление о текущем состоянии местного бюджета;

- экономическую значимость - практическое применение положений обеспечит поддержку процессов модернизации системы управления государственными финансами в виде определенного «мерила» качества принимаемых решений относительно схемы распределения региональных государственных финансовых потоков. Также данная методика может стать «фундаментом» национальной системы оценки региональной бюджетной системы, основанной на рейтингах местных бюджетов. Кроме того, потенциально может появиться необходимость уточнения или дополнения официального определения государственного бюджета в Республике Беларусь.

К заинтересованным лицам, которым целесообразно использовать нашу методику, относятся:

органы государственного управления Совет министров, Министерство экономики и Министерство финансов;

местные исполнительные и распорядительные органы - областные исполнительные комитеты, областные Советы депутатов; учебные и научные учреждения - университеты и институты;

независимые аналитические организации.

\section{Вычислительный модельный эксперимент по методике комплексной оценки местных бюджетов}

Предварительная апробация настоящей методики осуществлялась на примере утвержденного бюджета Брестской области на 2017 г. (табл. 5). Он содержит 30 наименований доходов бюджета и 49 наименований расходов.

Этап 1. Получение описательных оценок бюджета.

Оценка местного бюджета $O_{\partial}$ производится следующим образом. В доходной части все наименования поступлений закодированы, но в недостаточной степени. Например, поступление «Подоходный налог с физических лиц» необходимо раскрыть до подразделов, которые в классификаторе имеют ненулевые значения:

1) подраздел 01 - «Подоходный налог с физических лиц»;

2) подраздел 02 - «Подоходный налог с физических лиц, исчисленный с доходов, полученных от осуществления предпринимательской деятельности»;

3) подраздел 03 - «Подоходный налог с физических лиц, исчисленный с доходов в виде выигрышей (возвращенных несыгранных ставок), полученных физическими лицами от организаторов азартных игр юридических лиц Республики Беларусь»;

4) подраздел 04 - «Подоходный налог с физических лиц, исчисленный с доходов в виде процентов, полученных по банковскому вкладу (депозиту), по денежным средствам, находящимся на текущем (расчетном) банковском счете».

Следовательно, имеется промежуточное

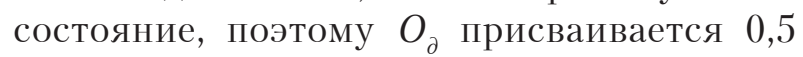
балла.

Оценка местного бюджета $O_{p}$ производится следующим образом. В расходной части бюджета все направления расходов закодированы в соответствии с классификатором и в полном объеме. Следовательно, $O$, присваивается 1 балл.

Общая описательная оценка $C_{o}$ в баллах вычисляется по формуле (1): 
Доходы и расходы областного бюджета Брестской области (укрупненные позиции), 2017 г.

\begin{tabular}{|c|c|c|c|c|c|}
\hline Поступления & $\begin{array}{c}\text { Код } \\
\text { поступлений } \\
\end{array}$ & Сумма, руб. & Расходы & $\begin{array}{c}\text { Код } \\
\text { расходов }\end{array}$ & Сумма, руб. \\
\hline Налоговые доходы & 1000000 & 441079217 & $\begin{array}{l}\text { Общегосударственная } \\
\text { деятельность }\end{array}$ & 010000 & 467161787 \\
\hline Налоги на доходы и прибыль & 1100000 & 212656145 & Национальная оборона & 020000 & 334119 \\
\hline Налоги на собственность & 1300000 & 18583040 & \multirow{4}{*}{$\begin{array}{l}\text { Судебная власть, } \\
\text { правоохранительная } \\
\text { деятельность и обеспечение } \\
\text { безопасности }\end{array}$} & \multirow{4}{*}{030000} & \multirow{4}{*}{9763973} \\
\hline Налоги на товары (работы, услуги) & 1400000 & 209667215 & & & \\
\hline Другие налоги & 1600000 & 172817 & & & \\
\hline Неналоговые доходы & 3000000 & 22714888 & & & \\
\hline $\begin{array}{l}\text { Доходы от использования имущества, } \\
\text { находящегося в государственной } \\
\text { собственности }\end{array}$ & 3100000 & 15655810 & Национальная экономика & 040000 & 170867624 \\
\hline $\begin{array}{l}\text { Доходы от осуществления } \\
\text { приносящей доходы деятельности }\end{array}$ & 3200000 & 5833668 & Охрана окружающей среды & 050000 & 361605 \\
\hline Штрафы, удержания & 3300000 & 20466 & $\begin{array}{l}\text { Жилищно-коммунальные услуги } \\
\text { и жилищное строительство } \\
\end{array}$ & 060000 & 97866780 \\
\hline Прочие неналоговые доходы & 3400000 & 1204944 & Здравоохранение & 070000 & 125964497 \\
\hline Безвозмездные поступления & 4000000 & 590718393 & \multirow{2}{*}{$\begin{array}{l}\text { Физическая культура, спорт, } \\
\text { культура и СМИ }\end{array}$} & \multirow{2}{*}{080000} & \multirow{2}{*}{27806043} \\
\hline Текущие дотации & 4316000 & 537095464 & & & \\
\hline Текущие субвенции & 4316100 & 80491322 & Образование & 090000 & 94278729 \\
\hline Капительные субвенции & 4326300 & 53622929 & Социальная политика & 100000 & 55168012 \\
\hline Итого & - & 1054512498 & Итого & - & 1049573169 \\
\hline
\end{tabular}

Источник. Решение Брестского областного Совета депутатов от 16 декабря 2016 г. № 216 «Об областном бюджете на 2017 год». Приложения 3, 4.

$C_{o}=1,5$ балла.

Этап 2. Получение расчетных оценок бюджета.

Оценка местного бюджета как плана формирования денежных средств $O_{\phi}$ рассчитывается по формуле $(2)^{11}: O_{\phi}=23 \%$. По оценочной шкале присвоен 1 балл.

Оценка местного бюджета как плана использования денежных средств состоит из 4 шагов.

Шаг 1. Анализ бюджета на наличие расхода «Бюджетные кредиты, ссуды, займы» код 011002.

Изначально областной бюджет Брестской области на 2017 г. был запланирован с превышением доходов над расходами в размере 4,9 млн руб. Расход «Бюджетные кредиты, ссуды, займы» по коду 011002 составил «минус» 8,6 млн руб.

В результате его исключения совокупные расходы изменились:

$$
Z=1049573169-(-8551429)=1058124598 \text { руб. }
$$

Превышение расходов над доходами стало равно 3612100 руб.

Шаг 2. Расчет матрицы «доход - расход» $X$ - пропорциональное распределение потоков при планировании бюджета.

По причине дефицитности утвержденного бюджета матрица $X$ рассчитывается по

$11 R=123 \%$ был вычислен по методике установления пороговых значений. первому варианту. В связи с большой размерностью матрицы $X$, где $(m+1) n=31 \cdot 49=$ $=1519$ ячеек, целесообразно привести образец расчета только для нескольких $x_{i j}$ по формуле (3).

1. Поступление «Подоходный налог с физических лиц» так распределяется на расход «Органы местного управления и самоуправления»:

$$
x_{1,1}=\frac{151067412 \cdot 11437761}{1058124598}=1632958 \text { руб. }
$$

2. Поступление «Налог на прибыль» распределяется на расход «Органы местного управления и самоуправления» следующим образом:

$$
x_{2,1}=\frac{59591646 \cdot 11437761}{1058124598}=644154 \text { руб. }
$$

и т. д.

3. Фиктивный доход с индексом $i=31 \quad$ в сумме дефицита бюджета в 3612100 руб. распределяется на расход «Другие вопросы в области социальной политики»:

$$
x_{31,49}=\frac{3612100 \cdot 17646318}{1058124598}=60239 \text { руб. }
$$

В табл. 6 в качестве примера приведена часть матрицы $X$ с распределенными вышеописанным образом потоками между 


\begin{tabular}{|c|c|c|c|c|c|c|c|c|c|c|c|c|c|c|c|c|c|c|}
\hline & 足 & 岕 & 용 & $i n$ & \pm & $\frac{\infty}{n}$ & $\begin{array}{l}n \\
\infty \\
\infty \\
n\end{array}$ & $\stackrel{\sim}{\varrho}$ & in & $\begin{array}{l}\stackrel{g}{0} \\
\stackrel{0}{E}\end{array}$ & 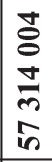 & 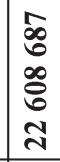 & $\begin{array}{l}\tilde{O} \\
\tilde{O} \\
\tilde{R} \\
\tilde{r}\end{array}$ & $\begin{array}{l}\tilde{O} \\
\infty \\
\infty \\
\infty \\
0\end{array}$ & 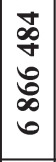 & $\mid \begin{array}{l}0 \\
\infty \\
\infty \\
\infty \\
\infty \\
1\end{array}$ & $\begin{array}{l}\text { 吉 } \\
\text { 号 } \\
\text { 号 } \\
-\end{array}$ & $\begin{array}{l}8 \\
0 \\
6 \\
6 \\
0\end{array}$ \\
\hline \multirow{14}{*}{ 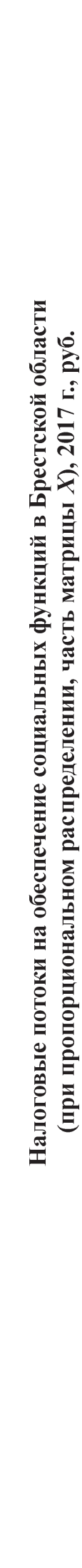 } & $\ddot{\infty}$ & 寽 & $\tilde{\check{\lambda}}$ & $\stackrel{\infty}{a}$ & ন & $\bar{\infty}$ & $\frac{\overline{0}}{0}$ & స్ & $a$ & $\vec{q}$ & 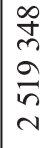 & $\begin{array}{l}\infty \\
\delta \\
\tilde{\alpha} \\
\delta\end{array}$ & $\begin{array}{l}n \\
\delta \\
\infty \\
\tilde{m} \\
\end{array}$ & 今ે & $\begin{array}{c}0 \\
\infty \\
\infty \\
\tilde{D} \\
\end{array}$ & $\mid \begin{array}{c}0 \\
\tilde{\sigma} \\
\stackrel{\sigma}{\sigma} \\
\dot{\sigma} \\
m\end{array}$ & $\mid \begin{array}{l}2 \\
0 \\
0 \\
0 \\
0\end{array}$ & $\mid \begin{array}{c}\infty \\
\infty \\
\sim\end{array}$ \\
\hline & 产 & 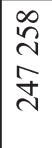 & $\begin{array}{l}0 \\
\tilde{n} \\
\hat{\alpha} \\
\end{array}$ & $\begin{array}{l}\text { iे } \\
\text { ch }\end{array}$ & $\grave{2}$ & $\begin{array}{l}\tilde{\Xi} \\
\text { సे }\end{array}$ & $\begin{array}{l}n \\
f \\
0 \\
n \\
n\end{array}$ & $\begin{array}{l}0 \\
\text { ñ }\end{array}$ & 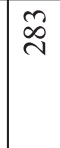 & 䎹 & $\begin{array}{l}8 \\
2 \\
\infty \\
2 \\
2 \\
-1\end{array}$ & $\begin{array}{l}\text { tr } \\
\stackrel{5}{\infty} \\
\infty \\
\infty \\
\end{array}$ & 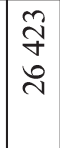 & 爱 & 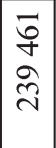 & 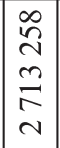 & $\begin{array}{l}\text { हे } \\
\infty \\
\text { ठ }\end{array}$ & $\begin{array}{l}\hat{\sim} \\
\text { ปे }\end{array}$ \\
\hline & $\stackrel{m}{m}$ & $\begin{array}{l}\tilde{r} \\
\tilde{f} \\
\tilde{n}\end{array}$ & $\begin{array}{l}\hat{\Sigma} \\
\hat{2} \\
\text { ma }\end{array}$ & $\begin{array}{l}\infty \\
\dot{q} \\
\end{array}$ & $\Xi$ & $\underset{\overbrace{}}{\stackrel{\curvearrowleft}{f}}$ & \begin{tabular}{|l}
$\infty$ \\
8 \\
8 \\
\& \\
\end{tabular} & 交 & $F$ & F & $\begin{array}{l}\infty \\
\infty \\
\infty \\
\infty \\
\infty\end{array}$ & $\begin{array}{l}\frac{\pi}{0} \\
\tilde{\sigma}\end{array}$ & $\begin{array}{l}\stackrel{\infty}{\mathfrak{n}} \\
\stackrel{0}{0}\end{array}$ & $\frac{0}{0}$ & $\begin{array}{l}n \\
a \\
\hat{a}\end{array}$ & \begin{tabular}{|l|} 
\\
0 \\
0 \\
0 \\
0 \\
0 \\
\end{tabular} & 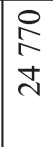 & $\bar{\sigma}$ \\
\hline & $\tilde{ల}$ & $\begin{array}{l}\vec{n} \\
0 \\
0 \\
2 \\
-\end{array}$ & 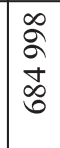 & 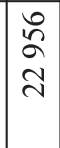 & $\begin{array}{l}\hat{\circ} \\
\text { nn }\end{array}$ & $\begin{array}{l}F \\
\text { O } \\
\stackrel{\infty}{0}\end{array}$ & $\begin{array}{l}\mathcal{N} \\
\stackrel{\sim}{\sim} \\
\tilde{n} \\
\sim \\
\sim\end{array}$ & $\begin{array}{l}n \\
\infty \\
i \\
n\end{array}$ & $\stackrel{\mathscr{\infty}}{2}$ & \% & $\begin{array}{l}\infty \\
\text { on }\end{array}$ & 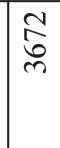 & $\tilde{\Xi}$ & i & $\stackrel{n}{\Xi}$ & 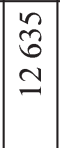 & 离 & $=$ \\
\hline & $\bar{\infty}$ & $\begin{array}{l}\tilde{8} \\
0 \\
0\end{array}$ & $\begin{array}{l}\text { શ } \\
\infty \\
\text { ని }\end{array}$ & $\begin{array}{l}0 \\
ٍ \\
\end{array}$ & స్ & $\underset{I}{\beth}$ & $\begin{array}{l}\tilde{\tilde{N}} \\
\tilde{n}\end{array}$ & 令 & $\stackrel{0}{=}$ & fo & $\begin{array}{l}\infty \\
0 \\
i n \\
n \\
n \\
\sim\end{array}$ & $\begin{array}{l}\text { t } \\
\text { O } \\
8 \\
8 \\
\end{array}$ & $\begin{array}{l}m \\
\tilde{n} \\
m \\
m\end{array}$ & $\frac{\infty}{\infty}$ & $\begin{array}{l} \pm \\
i \\
\tilde{n} \\
\tilde{n}\end{array}$ & $\mid \begin{array}{c}\tilde{O} \\
\stackrel{\sim}{\sim} \\
\vec{J} \\
m\end{array}$ & 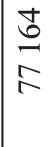 & ¿̀ \\
\hline & లి & $\begin{array}{l}n \\
2 \\
\tilde{y} \\
\infty \\
-\end{array}$ & 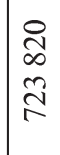 & 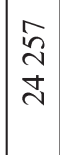 & $\begin{array}{l}\text { D } \\
\infty \\
\text { n. }\end{array}$ & $\begin{array}{l}\overline{\tilde{\infty}} \\
\hat{\partial} \\
\bar{\sim}\end{array}$ & 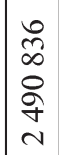 & $\begin{array}{l}\tilde{N} \\
\infty \\
\text { in }\end{array}$ & ڤેेે & F & 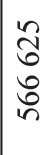 & $\begin{array}{l}\tilde{n} \\
\tilde{\lambda} \\
\tilde{N}\end{array}$ & $\overline{\frac{9}{7}}$ & $\frac{\sigma}{\infty}$ & $\begin{array}{l}0 \\
\infty \\
\infty \\
5\end{array}$ & $\begin{array}{l} \pm \\
\stackrel{ \pm}{2} \\
\stackrel{2}{r}\end{array}$ & 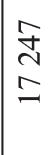 & 我 \\
\hline & ते & $\begin{array}{l}\tilde{m} \\
\tilde{\delta} \\
\hat{\sigma} \\
\tilde{2}\end{array}$ & $\begin{array}{l}\text { m } \\
\infty \\
\infty \\
\infty \\
\infty\end{array}$ & $\begin{array}{l}n \\
= \\
6\end{array}$ & के & $\begin{array}{l}0 \\
\text { O } \\
0 \\
0 \\
0 \\
\end{array}$ & \begin{tabular}{l}
$\hat{2}$ \\
\multirow{2}{0}{} \\
6 \\
0
\end{tabular} & $\frac{8}{n}$ & 恕 & 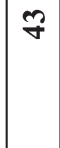 & $\begin{array}{l}\text { J } \\
\hat{N} \\
\sigma \\
\gamma\end{array}$ & $\begin{array}{l}\infty \\
\text { Oे } \\
\text { ปे } \\
\text { | }\end{array}$ & 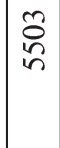 & $\begin{array}{l}n \\
m \\
m\end{array}$ & 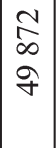 & $\mid \begin{array}{l}2 \\
0 \\
0 \\
0 \\
n\end{array}$ & $\begin{array}{l}\sqrt{6} \\
\simeq\end{array}$ & $\begin{array}{l}\stackrel{\circ}{\Im} \\
\stackrel{9}{*}\end{array}$ \\
\hline & $\stackrel{\infty}{\mathbf{a}}$ & $\begin{array}{l}0 \\
0 \\
0 \\
0 \\
\infty \\
-\infty\end{array}$ & $\begin{array}{l}\hat{n} \\
\infty \\
\approx \\
\approx\end{array}$ & 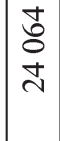 & $\begin{array}{l}\infty \\
\infty \\
\infty \\
n\end{array}$ & $\begin{array}{l}\bar{\infty} \\
\infty \\
\infty \\
\sim\end{array}$ & $\begin{array}{l}\tilde{8} \\
\text { 字 } \\
\text { r }\end{array}$ & 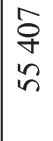 & 令 & $\mathcal{F}$ & $\begin{array}{l}\tilde{n} \\
\tilde{n} \\
\tilde{n} \\
\tilde{m}\end{array}$ & $\begin{array}{l}0 \\
\dot{b} \\
+ \\
0 \\
0 \\
0\end{array}$ & 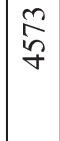 & $\stackrel{\circ}{\varrho}$ & $\frac{\nexists}{\forall}$ & $\mid \begin{array}{l}2 \\
\hat{n} \\
\hat{\delta} \\
+\end{array}$ & $\begin{array}{l}0 \\
\tilde{n} \\
0 \\
0\end{array}$ & ஓి \\
\hline & $\hat{\approx}$ & 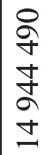 & $\begin{array}{l}\bar{\sigma} \\
\tilde{n} \\
\infty \\
\infty \\
n\end{array}$ & $\begin{array}{l}\mathbf{t} \\
2 \\
\hat{\sigma} \\
2\end{array}$ & \begin{tabular}{l}
$\stackrel{2}{\alpha}$ \\
\multirow{\gamma}{\gamma}{}
\end{tabular} & $\begin{array}{l}\stackrel{2}{\sigma} \\
\stackrel{2}{2} \\
\stackrel{2}{-}\end{array}$ & 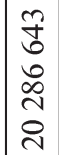 & $\begin{array}{l}\infty \\
\infty \\
\infty \\
+ \\
+ \\
y\end{array}$ & $\begin{array}{l}\text { ¿ } \\
\text { ○े } \\
-\end{array}$ & $F$ & $\begin{array}{l}\tilde{W} \\
\mathcal{N} \\
\infty \\
\infty \\
\tilde{m} \\
m\end{array}$ & 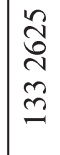 & $\begin{array}{l}8 \\
8 \\
7 \\
7\end{array}$ & $\begin{array}{l}+ \\
\infty \\
\infty \\
0\end{array}$ & $\begin{array}{l}\bar{r} \\
\dot{q} \\
\dot{q}\end{array}$ & $\begin{array}{l}\hat{a} \\
\infty \\
\infty \\
\infty \\
i n \\
\forall\end{array}$ & $\begin{array}{l}\text { సे } \\
\infty \\
\tilde{0} \\
\tilde{O}\end{array}$ & $\mid \begin{array}{l}n \\
\infty \\
\infty\end{array}$ \\
\hline & $\stackrel{i}{i}$ & $\begin{array}{l}n \\
\infty \\
\infty \\
n \\
\infty \\
\sim\end{array}$ & $\begin{array}{l}\tilde{N} \\
\tilde{N} \\
\beth\end{array}$ & $\begin{array}{l}\otimes \\
\infty \\
\tilde{n}\end{array}$ & $\frac{a}{a}$ & $\begin{array}{l}\tilde{N} \\
\tilde{N} \\
\tilde{\sim} \\
\tilde{J}\end{array}$ & 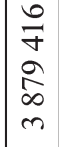 & $\begin{array}{l}\infty \\
\infty \\
0 \\
\infty\end{array}$ & iे & $q$ & 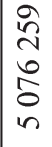 & $\begin{array}{l}n \\
\tilde{f} \\
\tilde{\sigma} \\
\tilde{\delta} \\
\delta \\
\sim\end{array}$ & $\frac{\mathfrak{0}}{\hat{\sigma}}$ & $\begin{array}{l}\stackrel{\hat{2}}{\hat{N}} \\
\underline{0}\end{array}$ & 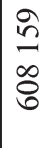 & $\mid \begin{array}{c}\vec{\infty} \\
\infty \\
8 \\
\infty \\
0\end{array}$ & $\begin{array}{l} \pm \\
n \\
\dot{n} \\
n\end{array}$ & $\begin{array}{l}\mathbf{D} \\
\stackrel{8}{n}\end{array}$ \\
\hline & $\mathscr{\sim}$ & 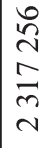 & $\begin{array}{l}\partial \\
o \\
\dot{\partial} \\
\sigma\end{array}$ & $\begin{array}{l}\mathbf{Z} \\
\text { } \\
0 \\
0\end{array}$ & $\overline{\tilde{g}}$ & 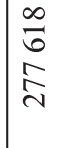 & $\begin{array}{l}\hat{\sigma} \\
\hat{n} \\
\mathfrak{n} \\
n \\
n\end{array}$ & $\begin{array}{l}+\tilde{n} \\
\tilde{n} \\
\stackrel{2}{r}\end{array}$ & 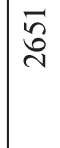 & లి & 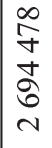 & $\begin{array}{l}\tilde{\delta} \\
\delta \\
\tilde{\delta} \\
\delta\end{array}$ & $\begin{array}{l}\overline{\widetilde{\sigma}} \\
n \\
n\end{array}$ & 守 & $\begin{array}{l}= \\
\bar{\infty} \\
\tilde{N} \\
\tilde{n}\end{array}$ & $\left|\begin{array}{l}0 \\
0 \\
0 \\
\tilde{n} \\
0 \\
m\end{array}\right|$ & $\begin{array}{l}0 \\
0 \\
0 \\
\infty\end{array}$ & 命 \\
\hline & $\mathbf{d}$ & $\begin{array}{l}\hat{2} \\
\hat{2} \\
\hat{2} \\
\infty\end{array}$ & 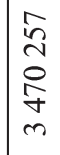 & $\mid \begin{array}{l}\infty \\
\text { iे } \\
\text { ర్ } \\
=\end{array}$ & $\begin{array}{l}\stackrel{\sim}{\sim} \\
\stackrel{\sim}{\sim}\end{array}$ & $\begin{array}{l}\tilde{\kappa} \\
\tilde{n} \\
\tilde{\delta} \\
-\end{array}$ & $\begin{array}{l}\hat{\sigma} \\
\exists \\
\Xi \\
=\end{array}$ & 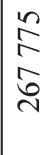 & $\begin{array}{l}\text { ț } \\
0 \\
0\end{array}$ & $\underset{ల}{\infty}$ & 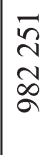 & $\begin{array}{l}\hat{\sigma} \\
\hat{\sigma} \\
\infty \\
\infty\end{array}$ & $\begin{array}{l}2 \\
2 \\
\approx \\
\simeq\end{array}$ & $\frac{\stackrel{n}{m}}{m}$ & $\begin{array}{l}\infty \\
\hat{\sigma} \\
ٍ \\
\Xi\end{array}$ & $\mid \begin{array}{c}m \\
\tilde{m} \\
\tilde{m} \\
\tilde{m}\end{array}$ & 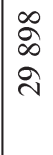 & $\stackrel{\Xi}{\Xi}$ \\
\hline & $\ddot{\sim}$ & $\frac{\pi}{n}$ & $\begin{array}{l}n \\
i \\
\tilde{2} \\
\delta\end{array}$ & 泃 & 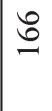 & $\frac{\infty}{6}$ & $\begin{array}{l}\overrightarrow{0} \\
\stackrel{0}{2} \\
\stackrel{2}{0}\end{array}$ & $\stackrel{\vec{n}}{2}$ & in & $\hat{m}$ & 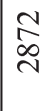 & $\stackrel{M}{=}$ & $\infty$ & $a$ & 导 & $\begin{array}{l}\infty \\
\infty \\
\infty \\
\text { en }\end{array}$ & $\infty$ & $m$ \\
\hline & $\therefore$ & - & $N$ & $\infty$ & $\nabla$ & in & b & $r$ & $\infty$ & $\approx$ & - & $N$ & $\infty$ & $\nabla$ & in & 0 & r & $\infty$ \\
\hline
\end{tabular}


налоговыми поступлениями и направлениями расходов на социальные нужды.

Iаг 3. Расчет матрицы «доход - расход» $Y-$ пропорциональные потоки при исполнении бюджета.

Анализ структуры и перечня доходов и расходов отчета об исполнении бюджета за 2017 г. показал, что имеются существенные отличия от утвержденного бюджета. Следовательно, необходимо привести эти матрицы к единообразному виду.

Так, выявлено превышение доходов над расходами в сумме 15081675 руб., которая была отнесена на фиктивный расход для балансировки бюджета в матрице $Y$ и внесена в матрицу $X$ с нулевым значением по фиктивному расходу. При этом фиктивный доход в матрице $X$ в размере 3612 100 руб. был внесен в матрицу $Y$ с нулевым значением. Дополнительно выявлено, что в разделе исполненных расходов «Общегосударственная деятельность» по кодам 010903 «Фонд финансирования расходов, связанных со стихийными бедствиями, авариями и катастрофами» и 010904 «Резервные фонды местных исполнительных и распорядительных органов» нет фактически понесенных расходов. Кроме того, в отчет об исполнении добавлен расход «Прочие отрасли национальной экономики» в сумме 4447950 руб., который был внесен в матрицу $X$ с нулевым значением. Таким образом, в матрицу $X$ добавлены два столбца по расходам, а в матрицу $Y$ - один столбец по расходам и одна строка по доходам (из матрицы $X$ ). В результате этих преобразований матрицы $X$ и $Y$ стали иметь равную размерность 32 на 51 ячейку, а также одинаковый перечень доходов и расходов. При этом баланс в матрицах не нарушен. После внесения всех изменений матрицы $X$ и $Y$ пересчитаны заново.

Шаг 4. Оценка местного бюджета как плана использования денежных средств $O_{u}$ рассчитывается по формуле (4):

$$
O_{u}=8,41 \% \text {. }
$$

Затем по шкале оценки (см. табл. 3) присваиваются баллы - присвоено 4 балла.

Прогнозная оценка рассчитывается по формуле 5: $O_{n}=91,59 \%$. В то же время при валовом сопоставлении исполненного и утвержденного вариантов бюджета отклонение по доходам составляет 101,3\%, а по расходам - 99,2\%. Таким образом, точность прогноза на стадии планирования не превышала 92\%, хотя при прямом (валовом) сопоставлении цифры более оптимистичные.

Общая расчетная оценка $C_{p}$ в баллах вычисляется по формуле (6):

$$
C_{p}=5 \text { баллов. }
$$

Этап 3. Получение комплексной оценки местного бюджета, присвоение класса бюджету.

Определяется комплексная оценка $O_{\kappa}$ по формуле (7):

\section{$O_{\kappa}=0,652$.}

Класс бюджету присваивается на основании табл. 4 - присвоен 3 класс (низкий).

По итогам оценки заполнен отчет (табл. 7).

Таблица 7

\section{Отчет об оценке}

\begin{tabular}{|l|c|}
\hline \multicolumn{1}{|c|}{ Показатель } & Значение \\
\hline Уровень бюджета & Областной \\
\hline АТЕ & Брестская область \\
\hline Год & 2017 \\
\hline Дефицит (-), профицит (+) & -3612100 \\
\hline Оценка $O_{\partial}$, балл & 0,5 \\
\hline Оценка $O_{p}$, балл & 1 \\
\hline Оценка $O_{\phi}, \% /$ балл & $23 / 1$ \\
\hline Оценка $O_{u}, \% /$ балл & $8,4 / 4$ \\
\hline Оценка $O_{\kappa}$ & 0,625 \\
\hline Класс & 3 \\
\hline
\end{tabular}

Источник. Авторская разработка.

Настоящая методика имеет определенные перспективы развития собственного потенциала. Так, возможна оценка бюджета не только на основе двухмерного представления данных вида «доход - расход», но и одновременным применением нескольких классификационных категорий расходов для получения многомерных потоков вида «доход - расход функциональный расход экономический - расход по ведомствам - расход по программам». Несмотря на то, что это потребует существенного усложнения используемого математического аппарата и увеличения вычислительных мощностей, оценивание местного бюджета 
на основе многомерных потоков может значительно увеличить ценность получаемой информации для последующего анализа.

Разработанная методика оценки местных бюджетов во многом носит экспериментальный характер и может дискутироваться. Обладая определенными достоинствами, она имеет ряд особенностей, игнорирование которых приведет к искажающей информации о состоянии бюджета. Так, целесообразно распределение направлений расходов безвозмездных поступлений: дотаций, субвенций и иных межбюджетных трансфертов. Также важно развитие по пути устранения перечисленных выше особенностей в части научного обоснования шкал оценки и классности, а также вовлечение в процесс оценивания информации о дефиците/профиците местного бюджета.

Предложенная методика просчитана на областном бюджете Брестской области на 2017 г. Бюджет был аттестован по 3 классу соответствия, что в целом хорошо согласуется с третьим местом Брестской области по объему ВРП за 2016-2018 гг. и с четвертым местом по объему ВРП на душу населения за тот же период ${ }^{12}$.

Оценивание местных бюджетов как способ измерения качества управления государственными финансами может внести существенный вклад в развитие региональных финансовых систем, что будет представлять определенный интерес для органов государственного управления и самоуправления на национальном и региональном уровнях, а также в научно-

12 Регионы Республики Беларусь, 2019: стат. сборник. 2019. Минск: Национальный статистический комитет Республики Беларусь. образовательной среде. Последующее развитие методики направлено на совершенствование или модификацию ее исходных положений для достижения новых преимуществ.

\section{СПИСОК ЛИТЕРАТУРЫ (REFERENCES)}

Сорокина Т.В. 1998. Концептуальные подходы к формированию межбюджетных отношений. Вестник Белорусского государственного экономического университета. № 2. С. 79-87. [Sorokina T.V. 1998. Conceptual approaches to the formation of interbudgetary relations. Vestnik Belorusskogo gosudarstvennogo ekonomicheskogo universiteta. No 2. PP. 79-87. (In Russ.)]

Сорокина T.B. 2020. Бюджетные риски: теория и методика оценки. Научные труды Белорусского государственного экономического универcumema. Вып. 13. С. 467-473. [Sorokina T.V. 2020. Budgetary Risks: Theory and Methodology of Assessment. Nauchnye trudy Belorusskogo gosudarstvennogo ekonomicheskogo universiteta. Iss. 13. PP. 467-473. (In Russ.)]

Тюлюндиева Н.M. 2011. Концепция управления бюджетом государства. Бишкек. 274 с. [Tiuliundieva N.M. 2011. State budget management concept. Bishkek. 274 p. (In Russ.)]

Шимов В.Н., Александрович Я.М., Богданович А.В., Ткачев С.П. (Ред.). 2001. Прогнозирование сочиально-экономического развития Республики Беларусь: вопросы теории и методики. Минск: НИЭИ Министерства экономики Республики Беларусь. 336 с. [Shimov V.N., Aleksandrovich Ya.M., Bogdanovich A.V., Tkachev S.P. (Eds.). 2001. Forecasting the socio-economic development of the Republic of Belarus: theory and methodology. Minsk: NIEI Ministerstva ekonomiki Respubliki Belarus'. 336 p. (In Russ.)]

Mutanov G. 2015. Mathematical Methods and Models in Economic Planning, Management and Budgeting. Berlin: Springer. 364 p. 
In citation: Belorusskiy Ekonomicheskiy zhurnal. 2021. No 3. PP. 113-124.

Belarusian Economic Journal. 2021. No 3. PP. 113-124.

\title{
COMPREHENSIVE ASSESSMENT OF LOCAL BUDGETS OF THE REPUBLIC OF BELARUS
}

\author{
Maksim Polovcev ${ }^{1}$ \\ Author affiliation: ${ }^{1}$ Economic Research Institute of the Ministry of Economy of the Republic \\ of Belarus (Minsk, Belarus). \\ Corresponding author: Maksim Polovcev (optimat@tut.by).
}

ABSTRACT. The article suggests a methodology for a comprehensive assessment of local budgets of the Republic of Belarus. Evaluation occurs by comparing the official quantitative characteristics of the budget. The score obtained is the basis for assigning a compliance class to the budget. Preliminary testing was carried out in the regional budget of the Brest region for 2017.

KEYWORDS: local budget, budget estimation, modeling.

JEL-code: H61, H72, R51.

DOI: $10.46782 / 1818-4510-2021-3-113-124$

Received 22.02.2021 